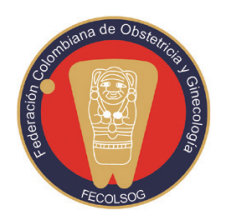

Revista Colombiana de Obstetricia y Ginecología Vol. 66 No. 1 • Enero-Marzo 2015 • (53-60)

ARtículo De REFLEXIÓN

DOI: http://dx.doi.org/10.18597/rcog.8

\title{
UNA APROXIMACIÓN DESDE LA FÍSICA A LAS CONSECUENCIAS PATOLÓGICAS DE LA LONGITUD EXCESIVA DEL CORDÓN UMBILICAL
}

\section{A physics approach to the consequences of excess unbilicall cord length}

\author{
Mercedes Olaya-Contreras, $M D^{1}$; William Vargas-Moreno ${ }^{2}$; \\ Jaime Eduardo Bernal-Villegas, $M D^{3}$
}

Recibido: mayo 26/14 - Aceptado: febrero 9/15

\section{RESUMEN}

Introducción: el cordón umbilical es una estructura incomprendida a pesar de transportar todo lo que un feto necesita para crecer y subsistir. Cada una de sus características es relevante, y la principal es la longitud, la cual, cuando está alterada, se relaciona con pobre pronóstico neurológico y muerte fetal.

Materiales y métodos: con base en la revisión de principios físicos, se proponen fórmulas que podrían explicar la importancia de la longitud del cordón por sus repercusiones hemodinámicas.

Resultados: se analizó la circulación fetal desde el corazón hasta la red capilar vellositaria en el plato placentario y, por medio de fórmulas físicas, se plantea la importancia de la longitud del cordón en la perfusión fetal.

Conclusiones: las complicaciones clínicas conocidas para la excesiva longitud del cordón umbilical fueron analizadas desde el punto de vista de la física, dejando listas las fórmulas para ser alimentadas

1 Médica Cirujana. Especialista en Patología. Pasantía en Patología Pediátrica. Estudiante de Doctorado en Ciencias Biológicas. Profesora Asistente, Departamento de Patología, Facultad de Medicina, Pontificia Universidad Javeriana, Hospital Universitario San Ignacio, Bogotá (Colombia).olaya.m@javeriana.edu.co; molayac@hotmail.com

2 Físico. Grupo de Física Forense, Instituto Nacional de Medicina Legal y Ciencias Forenses, Bogotá (Colombia).

3 Médico genetista. Profesor titular, Pontificia Universidad Javeriana, Instituto de Genética Humana, Facultad de Medicina, Pontificia Universidad Javeriana, Bogotá (Colombia). con datos reales provenientes de doppler fetales y mediciones del cordón umbilical.

Palabras clave: mortinato, cordón umbilical, trombosis, física.

\section{ABSTRACT}

Introduction: Despite its role of transporting all the things that a foetus needs to grow and survive, the umbilical cord is still poorly understood. All of its characteristics are relevant, and paramount among them is its length. When abnormal, length is associated with poor neurological prognosis and foetal demise.

Materials and methods: Based on a review of physics principles, formulae were proposed in an attempt to explain the importance of umbilical cord length on the basis of its haemodynamic repercussions.

Results: Foetal circulation was analysed from the heart to the placental villous capillaries and physics formulae were used to describe the importance of umbilical cord length in foetal perfusion.

Conclusions: Clinical complications known to be associated with excess umbilical cord length were analysed from the physics perspective, and formulae were established for use with real data derived from foetal Doppler scans and umbilical cord measurements. 
Key words: Stillborn, umbilical cord, thrombosis, physics.

\section{INTRODUCCIÓN}

La evolución ha permitido que el 85 \% de los mamíferos sean actualmente placentados, lo que corresponde a 4.486 especies (1); el cordón umbilical se ha adaptado a cada tipo de placenta, de útero y de cría (2). El cordón umbilical es una importante estructura de la que depende el suministro de nutrientes y de oxígeno del feto (3). Es aparentemente sencillo y por él discurren tres vasos que viajan cubiertos por la gelatina de Wharton, sin mayor protección para ser una extensión extracorpórea del sistema vascular fetal (4).

Por esto, no es extraño que situaciones que afecten al cordón umbilical, se reflejen en el feto y en la placenta y, además, pueden ser desde peligrosas hasta catastróficas: las alteraciones del cordón umbilical se consideran la causa de alrededor del $35 \%$ de los abortos espontáneos (5); el compromiso de la circulación fetal se sospecha en al menos el $20 \%$ de las autopsias de mortinatos $(6,7)$, y los accidentes del cordón umbilical causan el 28,8 \% de los casos de anoxia intrauterina (8).

La longitud se ha definido como la principal característica del cordón umbilical (9), porque cuando es anormal, tiene graves implicaciones en la salud del feto, en la adaptación del recién nacido y en el pronóstico neonatal (10); no obstante, las características de las que depende siguen siendo un enigma (11). Al revisar bases de datos no se encontró literatura relacionada con el tema, por lo que la aproximación desde la física podría ser novedosa para el estudio de esta estructura. En este artículo se propone la hipótesis de que la longitud excesiva del cordón conlleva cambios cardiovasculares fetales, entre ellos la hipertrofia ventricular fetal de mal pronóstico.

El objetivo de este artículo, como primer paso para la comprobación de nuestra hipótesis, es proponer los fundamentos que, desde la física, permiten analizar el impacto de la longitud del cordón umbilical en la circulación fetal, lo que haría posible el abordaje del problema clínico de una manera más exacta, para un mejor entendimiento de las repercusiones de la modificación de la longitud del cordón umbilical en las condiciones fetales intrauterinas.

Se construyen unas fórmulas adaptadas al funcionamiento del corazón fetal humano y su bombeo hasta y desde los capilares en las vellosidades coriales, con base en principios físicos, buscando tener las bases para demostrar de manera teórica la implicación de la longitud en este proceso, que afecta al feto, al neonato y, eventualmente, al individuo más adelante en su desarrollo. Primero revisaremos la estructura y las funciones del cordón umbilical, los principios físicos que rigen la dinámica de los líquidos en el mismo, la fisiología cardiaca fetal y las consecuencias de la longitud del cordón sobre la función cardiovascular fetal. Se espera que en el futuro, a partir de las fórmulas físicas propuestas, se pueda evaluar clínicamente el efecto de la longitud del cordón con la perfusión cerebral fetal y umbilical examinada mediante doppler.

\section{ESTRUCTURA FÍSICA DEL CORDÓN Y SUS FUNCIONES}

El cordón umbilical es una estructura única por permitir la comunicación del feto con la placenta e indirectamente con la madre, y por transportar las sustancias esenciales requeridas por el hijo para mantener la vida y lograr su crecimiento y desarrollo $(12,13)$.

La principal función del cordón umbilical es el transporte de fluidos, los cuales son esenciales para el desarrollo y crecimiento del feto. En comparación, los vasos del cuerpo adulto tienen distribuidas las funciones de transporte: las arterias transportan sangre oxigenada, y las venas sangre no oxigenada. Las arterias constan de capa elástica interna y externa, y la vena únicamente de capa elástica externa (2). Los vasos del cordón, sin embargo, tienen esta función al revés, el transporte de sangre oxigenada se realiza por la vena y el de la sangre no oxigenada por las arterias. Las arterias del cordón no tienen capa elástica interna y la capa externa es 
discontinua (mucho más tenue que la del resto de las arterias del cuerpo) y, por el contrario, la vena tiene una capa elástica interna $(7,14)$. Tanto la vena como las arterias del cordón carecen de inervación (15). Con estas características, los vasos del cordón responden a la presión y al flujo que contienen; la gelatina de Wharton es esencial porque permite que el vaso aumente o disminuya su diámetro según el gasto. La gelatina de Wharton concede resistencia a presiones altas protegiendo a los vasos del colapso (16); así como el amnios que la circunda, el cual ayuda a regular la presión hidrostática; en el cordón el amnios está firmemente adherido, a diferencia del amnios de las membranas ovulares, el cual tiene una unión virtual al corión. El colapso vascular también se evita gracias al enrollamiento normal que presenta el cordón, el cual ocurre por partes, de las dos arterias sobre la vena y va hacia la izquierda (17-21).

\section{FÍSICA DE LA DINÁMICA DE LÍQUIDOS DEL CORDÓN UMBILICAL}

Desde el punto de vista físico, la resistencia a los fluidos que viajan dentro de un tubo depende de tres características básicas: la longitud y el diámetro del tubo, y la viscosidad del líquido. Los "tubos perfectos" son rígidos, rectos y de diámetros constantes, $y$, en un fluido ideal, la viscosidad debe ser cero; el resultado de estas condiciones es que el líquido no se adhiere a las paredes y no disipa energía. En los seres vivos, estas características no se presentan y, especialmente en el cordón umbilical, tenemos diferencias de diámetros, distintas longitudes, vasos normal y anormalmente enrollados, adelgazamientos, sitios sin protección de la gelatina de Wharton, sitios propensos a la estasis (nudos verdaderos y falsos), sitios de obstrucción (nudos verdaderos y estrecheces) (figura 1); además, la viscosidad puede

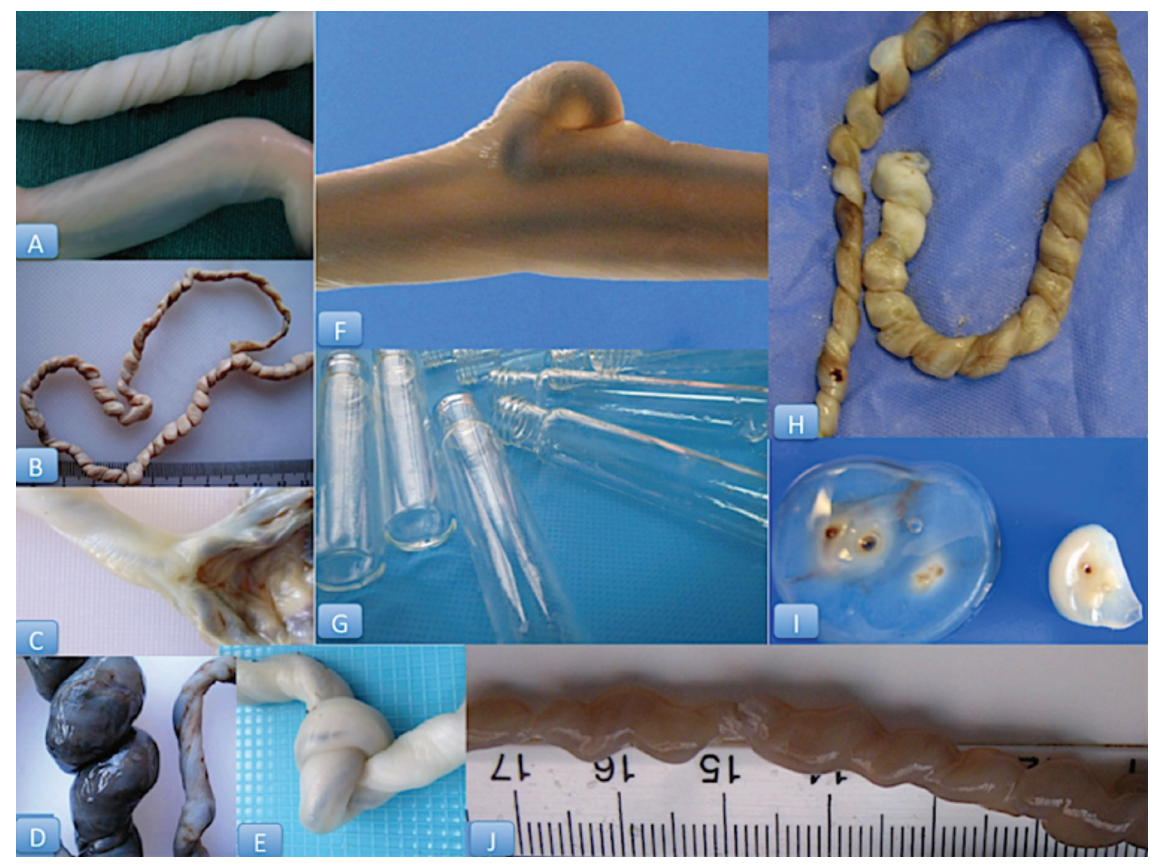

Figura 1. Tubos perfectos y "tubos reales"

A. Comparación entre hiper (arriba) e hipoenrollamiento (abajo) (ambos anormales). B. Cordón umbilical largo e hiperenrollado. C. Inserción anormal: furcata (los vasos pierden la protección de la gelatina de Wharton antes de entrar al parénquima velloso) y, además, marginal (llegan al borde, siempre mal perfundido, de la placenta). D. Comparación entre diámetros excesivo (izquierda) y deficiente (derecha) (ambos anormales). E. Nudo verdadero. F. Nudo falso. G. Tubos perfectos. H. Cordón umbilical largo e hiperenrollado. I. Comparación entre diámetros aumentado (izquierda) y reducido (derecha) (ambos anormales). J. Cordón con estrecheces (junto al 15 y al 17 de la regla). 
variar ante condiciones fisiológicas o patológicas. En la gestación, estos "inconvenientes" deben superarse para obtener el gasto preciso.

El gasto (Q) depende de que en un extremo del tubo haya una presión (P1) y en el otro extremo, otra diferente (P2). Se requiere que las presiones sean distintas, siendo mayor la presión que ingresa $(\mathrm{P} 1>\mathrm{P} 2)$, luego es detectable una diferencia entre presiones (P1 - P2). La resistencia (R), según la revisión de resistencia al flujo, puede expresarse con la siguiente ecuación (a):

$$
R=\frac{P 1-P 2}{Q}
$$

La resistencia (para flujo laminar) depende de la longitud (L), la viscosidad (M) y el radio (r); al considerar un fluido viscoso pero con flujo laminar, se puede expresar así (b):

$$
\mathrm{R}=\frac{8 L M}{\pi \mathrm{r}^{4}}
$$

En caso de un flujo laminar, R depende de L, de la viscosidad y del radio del tubo, de acuerdo con la expresión anterior.
Los cálculos de la resistencia podrían realizarse con cierta certeza si las variables cambiaran una por una, pero en la realidad no ocurre así; la longitud varía según el número de hijos, el tamaño uterino y otros factores dentro de rangos definidos como normales o anormales (3). Por su parte, la viscosidad puede cambiar rápidamente, por ejemplo, con la temperatura. El radio de los vasos es poco controlable dadas las características descritas de las que depende.

Así, podría pensarse que dentro de unos parámetros relativamente constantes, cada individuo manejaría su propia resistencia, la cual tiene varias formas de adaptarse a las circunstancias placentarias y fetales.

Para el flujo laminar de un fluido de viscosidad constante, se forma una discreta capa adyacente a las paredes que no se mueve, y hacia el centro del vaso se forman sucesivas capas que van aumentando su velocidad (en sentido externo-interno dentro del vaso) (18); entre cada capa y su vecina, se crea fricción, lo cual implica que se disipa energía (figura 2). La energía que se pone al principio del tubo debe ser suficiente para que, a pesar de que esta se disipe, el flujo llegue exitosamente al otro lado; por tanto, si el tubo es de mayor longitud, necesitará mayor

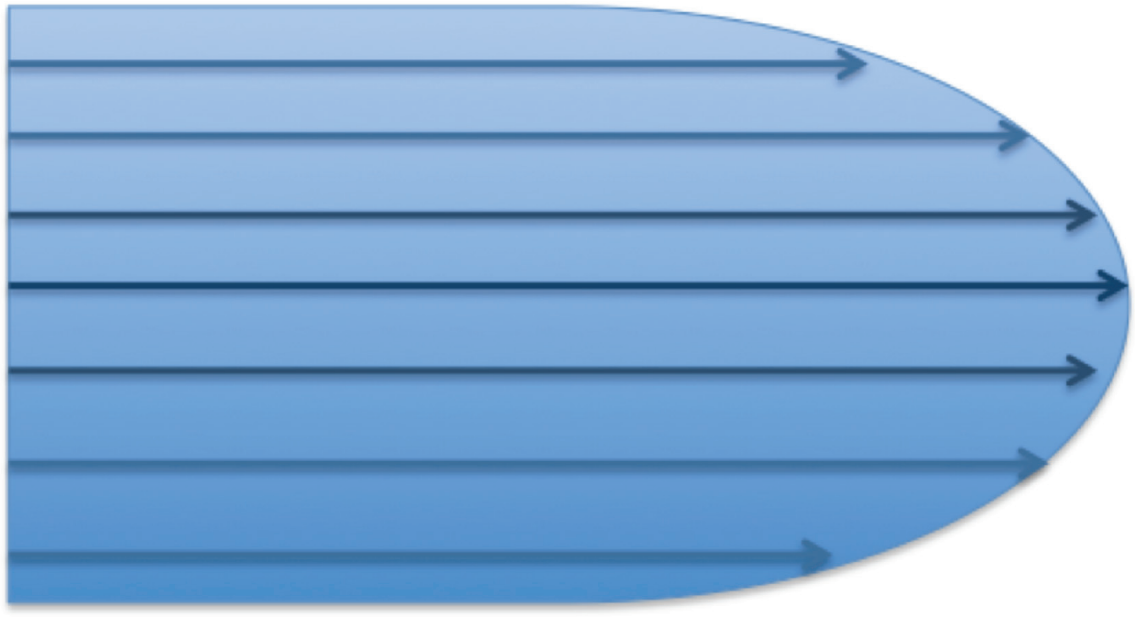

Figura 2. Flujo laminar

Hacia el centro del vaso se forman sucesivas capas que van aumentando su velocidad (en sentido externo-interno del vaso). 
cantidad de energía, en otras palabras, a mayor longitud, mayor energía disipada. Esto implica que para mantener un flujo constante hay que aumentar la energía y así conservar la diferencia de presiones entre los extremos del tubo. El responsable de la energía que se pone al inicio del sistema, o sea el responsable de mantener esta diferencia de presiones, es el corazón fetal.

\section{FISIOLOGÍA DE LA CIRCULACIÓN FETAL}

La circulación fetal es un circuito cerrado desde la vellosidad terciaria placentaria hasta las cavidades cardiacas fetales. En la placenta inician las vellosidades terciarias que contienen sangre recién oxigenada que es succionada en la diástole cardiaca fetal con gran participación de los movimientos diafragmáticos; esta asciende por las múltiples ramificaciones hasta la vena umbilical, tan larga como sea el cordón mismo, e ingresa al cuerpo fetal, a su sistema venoso, convirtiéndose en sangre mezclada; después pasa a través del ducto venoso en el hígado (figura 3) y se une con la vena cava inferior; este conducto es corto y angosto (además de temporal), lo que le imprime velocidad a la circulación, además es

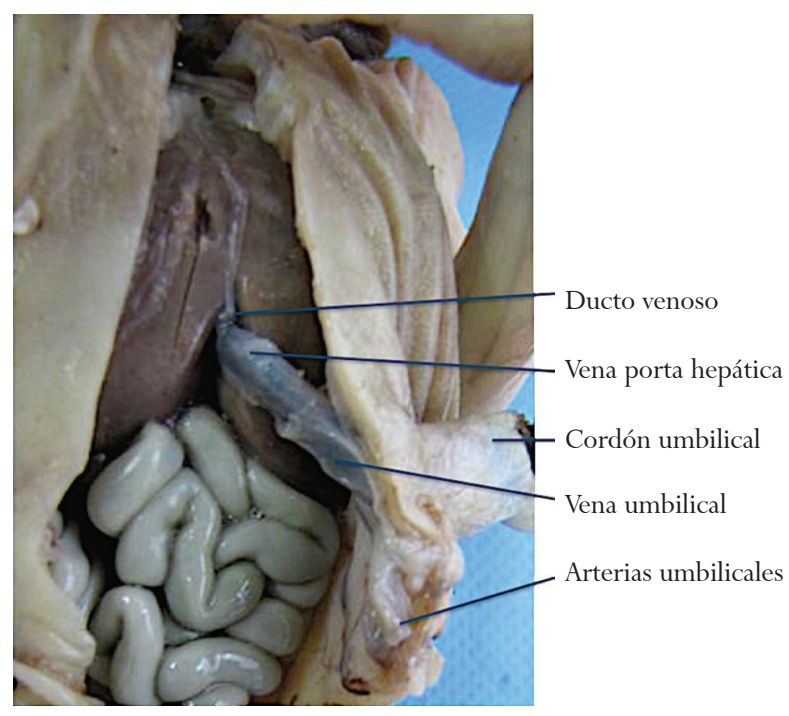

Figura 3. Entrada de los vasos umbilicales y su conexión con la circulación fetal.

Feto humano del segundo trimestre de la gestación. Muerte natural. un esfínter que impide que la sangre procedente de la porción inferior del cuerpo entre en la vena umbilical. Posteriormente, la sangre mezclada, una vez oxigenado el hígado, llega a la aurícula derecha al igual que la sangre venosa que llega por la cava superior. En este punto el flujo es pulsátil y turbulento (19).

Esta sangre, aunque mezclada, es la mejor oxigenada que maneja el feto. De ella pasa un $46 \%$ por el foramen oval a la aurícula izquierda asegurando que la circulación coronaria y cerebral supla su mayor demanda de oxígeno. Otra parte de la sangre de la aurícula derecha pasa al ventrículo derecho, sale por la arteria pulmonar hacia los pulmones (apenas entre 4 y $15 \%$ ); lo demás pasa de la arteria pulmonar por el ducto arterioso a la aorta descendente para su distribución en el resto del cuerpo.

Para el cierre del circuito, la sangre mezclada de la aorta descendente y las arterias iliacas, llega a las arterias umbilicales que llevan la sangre desoxigenada a las ramificaciones sucesivas del sistema vellositario en el plato basal placentario, hasta la vellosidad terciaria donde, a través de las membranas vásculo-sincitiales, se produce nuevamente el intercambio con la sangre materna (figura 4).

Hay dos circulaciones paralelas: la intrafetal y la placentaria, ellas se comunican indirectamente, sin mezclarse; la "circulación de intercambio" es la que viaja a través del cordón umbilical.

\section{CONSECUENCIAS DE LA LONGITUD DEL CORDÓN UMBILICAL EN LA FISIOLOGÍA FETAL}

El corazón bombea la sangre imprimiendo la presión necesaria para que esta recorra el circuito, gracias a las diferencias de presiones al principio y al final del tubo; pero el corazón tiene un límite en la entrega de la energía que garantiza tal diferencia de presiones. La energía que se necesita para este funcionamiento se afecta si la longitud del tubo aumenta, ya que se requeriría mayor energía. Cuando la longitud excede ciertos parámetros, puede llevar 


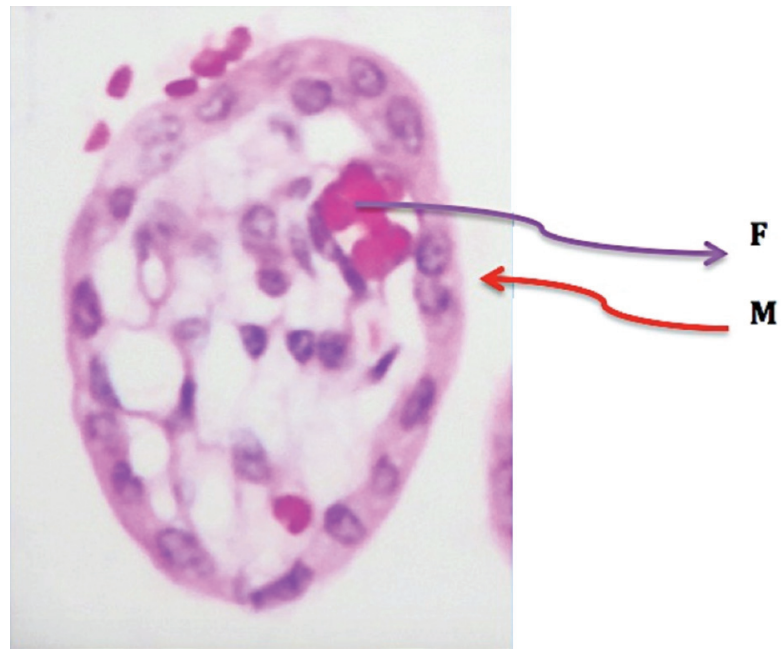

Figura 4

Vellosidad corial humana del tercer trimestre Membrana vásculo-sincitial Hematoxilina-Eosina 16x Circulación fetal (F) y circulación materna (M)

a insuficiente gasto, estasis sanguínea con el riesgo de eventos trombóticos y, ante mayor detrimento en el gasto, hay riesgo de muerte.

Clínicamente se sabe que cordones largos están asociados con complicaciones fetales $(4,20,21)$ tales como circulares al cuello o a otras partes del cuerpo fetal, excesivo enrollamiento, enrollamiento a la derecha (enrollamiento contrario), nudos verdaderos, arteria única, trombosis fetal, hipoxia, alteraciones del flujo placentario, imágenes cerebrales anormales (22) o anormalidades en el seguimiento neurológico $(23,24)$, cardiomegalia, hipoxia intrauterina, restricción del crecimiento intrauterino, muerte fetal, estado fetal insatisfactorio y vasculopatía trombótica fetal (25).

Como se dijo, la resistencia depende de las diferencias de presiones y del gasto, lo que nos lleva a la relación del gasto con la resistencia (c),

$$
R=\frac{P 1-P 2}{Q} \quad \mathrm{O} \quad \mathrm{Q}=\frac{P 1-P 2}{R}
$$

El gasto dentro del circuito depende en gran medida de la resistencia, y esta de los tres factores mencionados, siendo la longitud el factor más variable y el que clínicamente más se asocia con mal pronóstico fetal.

En los casos en los que hay un flujo turbulento y no laminar, la energía se disipa con mayor rapidez, por lo que la longitud sería un factor aún más determinante en contra del adecuado gasto. Considerando (d):

$$
Q=\frac{(P 1-P 2) \pi r^{4}}{8 L M}
$$

puede afirmarse que el gasto (Q) es una función de la longitud y (e). En este caso, un aumento en la longitud necesariamente disminuye el gasto.

$$
Q L \approx \text { Constante }(\mathrm{e})
$$

En condiciones reales de la circulación de un feto, tal afirmación no es certera, ya que se carece de los datos de estas variables (gasto real vs. longitud del cordón umbilical); por tanto, las reflexiones sobre las implicaciones clínicas ya conocidas de las condiciones físicas del cordón umbilical en el gasto cardiaco fetal $(9,11,26)$ se están materializando mediante aproximaciones sucesivas (expansiones de Q).

La alimentación de las fórmulas es la segunda parte de la investigación, donde mediante estudios de doppler fetal se están obteniendo datos de la circulación fetal (arteria cerebral media y arteria umbilical) y se están cotejando con la medición respectiva y sucesiva de cordones umbilicales completos, realizada en el Departamento de Patología del Hospital Universitario San Ignacio.

\section{CONCLUSIONES}

El cordón umbilical analizado desde la física, muestra una estructura contraria al "tubo ideal", y lleva un flujo que frecuentemente deja de ser laminar. Los vasos que suplen al feto están expuestos al viaje desde la placenta hasta el feto. El corazón fetal es el responsable de bombear, impulsando y absorbiendo en un circuito cerrado. 
El cordón es una extensión del sistema cardiovascular fetal y su longitud es un factor que afecta al gasto cardiaco. Desarrollamos las fórmulas físicas que, alimentadas con valores reales, nos mostrarán una mayor aproximación del impacto de la longitud del cordón umbilical en la perfusión fetal.

\section{FINANCIACIÓN}

El desarrollo completo de este trabajo está financiado por la Vicerrectoría de Investigación de la Pontificia Universidad Javeriana, con el concurso del Hospital Universitario San Ignacio.

\section{REFERENCIAS}

1. Ji Q, Luo ZX, Yuan CX, Wible JR, Zhang JP, Georgi JA. The earliest known eutherian mammal. Nature. 2002;416:816-22.

2. Wildman DE, Chen C, Erez O, Grossman LI, Goodman M, Romero R. Evolution of the mammalian placenta revealed by phylogenetic analysis. Proc Natl Acad Sci USA. 2006;103:3203-8.

3. Wang Y, Zhao S. Placental Blood Circulation. En: Wang Y, Zhao S. Vascular Biology of the Placenta. San Rafael (CA): Morgan \& Claypool Life Sciences; 2010. p. 3-11.

4. Lewis S, Benirschke K. Placenta. En: Mills S. Histology for Pathologists. Third edition. Philadelphia: Lippincott Williams and Wilkins; 2007. p. 1095-126.

5. Blackburn W. Umbilical cord. In: Stevenson RE, Hall JG. Human malformation and related anomalies. 2nd ed. New York: Oxford University Press; 2006. p. 1413-95.

6. Tantbirojn P, Saleemuddin A, Sirois K, Crum C, Boyd T, et al. Gross Abnormalities of the umbilical cord: Related Placental Histology and Clinical Significance. Placenta. 2009;30:1083-8.

7. Horn LC, Langner A, Stiehl P, Wittekind C, Faber R. Identification of the causes of intrauterine death during 310 consecutive autopsies. Eur J Obstet Gynecol Reprod Biol. 2004;113:134-8.

8. Collins JH, Collins CL, Collins CC. Silent risk - Issues about the Human Umbilical Cord. Umbilical cord accidents; 2010. [Visitado 2012 Nov 10]. Disponible en: http://www.preginst.com/pucp.html

9. Stefos T, Sotiriadis A, Vasilios D, Tsirkas P,Korkontzelos
I, Avgoustatos F. et al. Umbilical cord legth and paritythe Greek experience. Eur J Obstet Gynecol Reprod Biol. 2003;107:41-4.

10. Benirschke K, Burton G, Baergen R. Anatomy and pathology of the umbilical cord. En: Bernirschke K, Burton G, Baergen R. Pathology of the human placenta. 6th ed. New York: Springer; 2012. p. 309-76.

11. Olaya CM, Bernal JE. Comprendiendo el cordón umbilical. Patología Rev Latinoam. 2013;51:200-5.

12. Donlon CR, Furdon SA, Clark DA. Look before you clamp: delivery room examination of the umbilical cord. Adv Neonatal Care. 2002;2:19-26.

13. Ferguson VL, Dodson RB. Bioengineering aspects of the umbilical cord. Eur J Obstet Gynecol Reprod Biol. 2009; 144:S108-13.

14. Ernst L. Placenta. En: Ernst L, Ruchelli E, Huff DS, editors. Color Atlas of Fetal and Neonatal Histology. Chicago: Springer; 2011. p. 363-88.

15. Baergen RN. Pathology of the Umbilical Cord in Baergen RN. Manual of and Kaufmann's Pathology of the Human Placenta. Second Edition. New York: Springer; 2011. p. 247-80.

16. Kraus FT, Redline R, Gersell D, Nelson M, Dicke J. Anatomy, Structure, Development, and function. Placental Pathology. Washington, DC: American Registry of Pathology, Atlas of nontumor pathology, First series, Fascicle 3; 2004. p. 1-22.

17. Kalish R, Hunter T, Sharma G, Baergen R. Clinical significance of the umbilical cord twist. Am J Obstet Gynecol. 2003;189:736-9.

18. Hernández E, Benavides-Serralde, Cruz-Martínez R, Gratacós E. Conceptos esenciales sobre las bases físicas del Doppler para su utilización clínica. En: Gratacós E, Figueras F, Hernández E, Puerto B. Doppler en Medicina Fetal. Madrid: Editorial Médica Panamericana; 2010. p. 1-32.

19. Mortera C. Ecocardiografía fetal. Anatomía y fisiología normal del feto. En: Protocolos diagnósticos y terapéuticos en cardiología pediátrica. Unidad de Cardiología Pediátrica, Hospital San Juan de Dios, HCP. Barcelona. [Visitado 2013 Nov 15]. Disponible en: https://www.aeped.es/sites/default/ files/documentos/37_eco_fetal.pdf 
20. Balkawade NU, Shinde MA. Study of Length of Umbilical Cord and Fetal Outcome: A Study of 1,000 Deliveries. J Obstet Gynaecol India. 2012;62:520-5.

21. Chan JS, Baergen RN. Gross umbilical cord complications are associated with placental lesions of circulatory stasis and fetal hypoxia. Pediatr Dev Pathol. 2012;15:487-94.

22. Grafe MR. The correlation of prenatal brain damage with placental pathology. J Neuropathol Exp Neurol. 1994;53:407-15.

23. Baergen R. Cord abnormalities, structural lesions, and cord “accidents”. Semin Diagn Pathol. 2007;24:23-32.
24. Baergen RN, Malicki D, Behling C, Benirschke K. Morbidity, mortality, and placental pathology in excessively long umbilical cords: retrospective study. Pediatr Dev Pathol. 2001;4:414-53.

25. Redline RW. Clinical and pathological umbilical cord abnormalities in fetal thrombotic vasculopathy. Hum Pathol. 2004;35:1494-8.

26. Taweevisit M, Thorner PS. Massive fetal thrombotic vasculopathy associated with excessively long umbilical cord and fetal demise: case report and literature review. Pediatr Dev Pathol. 2010;13:112-5. 